\title{
Who's on First? Split and Shared Services in the NICU
}

Scott D. Duncan, MD, MHA

The baseball analogy is appropriate in neonatology, as the provision of neonatal care is a team sport, including, but not limited to, physicians, advanced practice providers, nursing, nutritionists, pharmacists, respiratory therapists, speech therapists, physical therapists, and social workers. In many instances, the neonatologist's role has undergone significant modifications, with an increasing role of advanced practice providers, as well as a reduction in trainee rotations and restrictions on work hours.

"In many instances, the neonatologist's role has undergone significant modifications, with an increasing role of advanced practice providers, as well as a reduction in trainee rotations and restrictions on work hours."

\section{Scenario}

In the batter's box, the delivery team attends the delivery of a 32week gestation infant due to maternal gestational diabetes and pre-eclampsia. The delivery team includes two nurses, a respiratory therapist, and a neonatal nurse practitioner (NNP) as the team leader. In the delivery room, the infant requires intubation and positive pressure ventilation. The infant is admitted to the NICU on a ventilator. By 30 minutes of age, the infant demonstrates increasing respiratory distress, hypercarbia, and the CXR reveals severe RDS. The NNP orders surfactant therapy.

While making rounds with the fellow and resident team, the neonatologist asks the NNP about the patient. The NNP gives the neonatologist a verbal report and completes the electronic medical record's history and physical (EMR). Following rounds, the neonatologist examines the infant and places a macro-based attestation used for resident documentation on the H\&P, co-signs the note, and uses the charge capture feature on the EMR to place a 99468 code for the date of admission.

\section{A Strikeout}

Strike One - The neonatologist provided limited services in the assessment and care plan.

Strike Two - A NNP is not a trainee, and Physician at Teaching Hospital (PATH) guidelines do not apply for documentation and attestations.

Strike Three - The service for the day is an initial critical care charge, and split/shared services are prohibited with critical care delivery.

\section{Discussion}

In this Scenario, the neonatologist made multiple errors as related to split/shared services and supervision. A split/shared service is an encounter where a physician and the NNP from the same group and same specialty each personally perform a portion of an Evaluation and Management (E/M) visit. As applied to in-patient neonatology, the split/shared $E / M$ rule applies only to selected $\mathrm{E} / \mathrm{M}$ visits such as these in the hospital settings:
- $\quad$ hospital admissions (CPT ${ }^{\circledR}$ codes 99221-99223)

- $\quad$ subsequent hospital visits (CPT ${ }^{\circledR}$ Codes 99231-99233)

- $\quad$ discharge management (CPT ${ }^{\circledR}$ Codes 99238-99239).

In the case of critical care, split/shared services are not allowed. The guidance provided by the US Department of Health and Human Services states, "Critical care services are reflective of the care and management of a critically ill or critically injured patient by an individual physician or qualified non-physician practitioner for the specified reportable period of time. Unlike other E/M services where a split/shared service is allowed, the critical care service reported shall reflect the evaluation, treatment, and management of a patient by an individual physician or qualified non-physician practitioner [NPP] and shall not be representative of combined service between a physician and a qualified NPP." (1)

Appropriate documentation must substantiate the nature of the admission. Per the recommendations of the AAP Committee on Coding and Nomenclature, (2) the medical record documentation should include:

- Documentation of the critical status of the infant

- Documentation of the bedside direction and supervision of all aspects of care

- Review of pertinent historical information and verification of significant physical findings through a medically indicated, focused patient examination

- Documentation of all services provided by members of the care team and discussion and direction of the ongoing therapy and plan of care for the patient

- Additional documentation of any major change in a patient course requiring significant hands-on intervention by the reporting provider.

\section{"Split/shared services may be utilized} for E/M services, hospital admissions, subsequent hospital visits, and discharge, as noted above. In these instances"

Split/shared services may be utilized for E/M services, hospital admissions, subsequent hospital visits, and discharge, as noted above. In these instances, the providers should each document their portion of the service provided. Charges may be placed using the following rules: (3)

- When the physician provides any face-to-face portion of the encounter, use either provider's NPI

- When the physician does not provide a face-to-face encounter, use the NNP's NPI.

Note, there is no guidance for split/shared services for intensive care charges $\left(\mathrm{CPT}^{\circledR}\right.$ codes 99477-99480). Best advice - if using a physician NPI for billing, ensure that the documentation clearly demonstrates that the physician took ownership of the patient.

\section{Revised Scenario - a pinch hitter}

At the plate, the delivery team attends the delivery of an infant of 32-week gestation due to maternal gestational diabetes and pre- 
eclampsia. The delivery team includes two nurses, a respiratory therapist, and a neonatal nurse practitioner (NNP) as the team leader. In the DR, the infant requires PPV and intubation. The infant is brought to the NICU on assisted ventilation. By 30 minutes of age, the infant demonstrates increasing respiratory distress, hypercarbia, and the CXR reveals severe RDS.

While making rounds with the fellow and resident team, the neonatologist asks the NNP about the patient. The NNP gives the neonatologist a verbal report, and the neonatologist leads the rounding team to the patient's bedside, where a complete examination is performed. The neonatologist orders surfactant replacement, and following rounds completes a history and physical, including the assessment and plan, in the EMR, independent of documentation by the NNP. The neonatologist uses the charge capture feature on the EMR to place a 99468 charge for the date of admission.

\section{A Home Run!}

\section{References:}

1. US Department of Health \& Human Services. Medicare Claims Processing Manual. In:30.36.12.E.32.

2. COCN American Academy of Pediatrics. Coding for Pediatrics 2021. Itasca, IL: American Academy of Pediatrics; 2021.

3. Centers for Medicare \& Medicaid Services. Evaluation and Management Services Guide. In:MLN906764:21.

Disclosure: The author has no disclosures.

NT

Corresponding Author:
Scott D. Duncan, MD, MHA.
Professor and Chief
Division of Neonatal Medicine
University of Louisville
571 S. Floyd St.
Suite 342
Louisville, KY 40202
P:502-852-8470
F:502-852-8473
sddunc02@louisville.edu

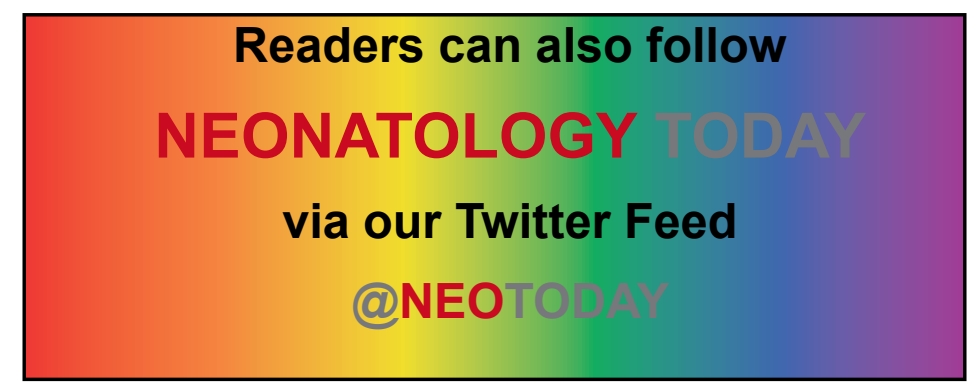

\section{Keeping vour Bably sate}

\section{during the COVID-19 pandemic}

\section{How to protect your little one from germs and viruses}

Even though there are some things we don't know about COVID-19 yet. there are many more things that we do know. We know that there are proven protective measures that we can take to stay healthy.

\section{Here's what you can do...}
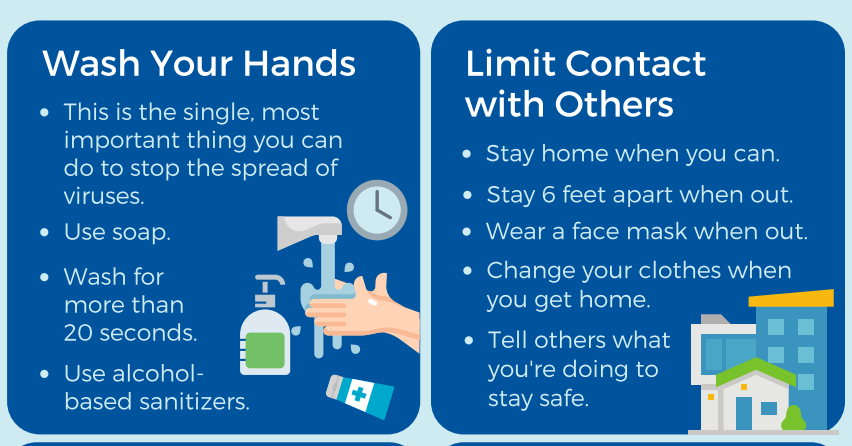

Provide P
Immunity

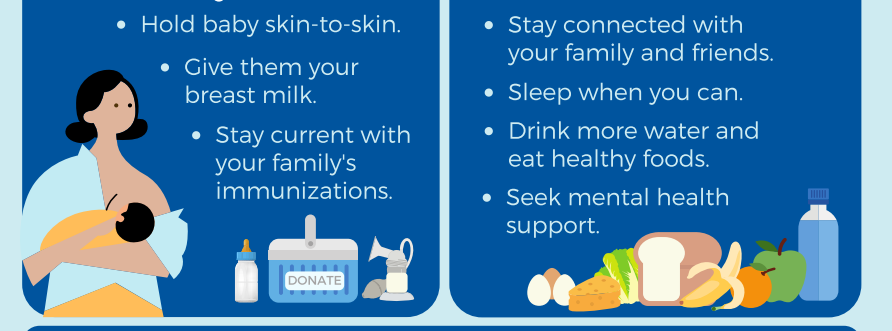
Immunizations Vaccinations save lives. Protecting your baby from
flu and pertussis lowers their risks for complications from coronavirus.
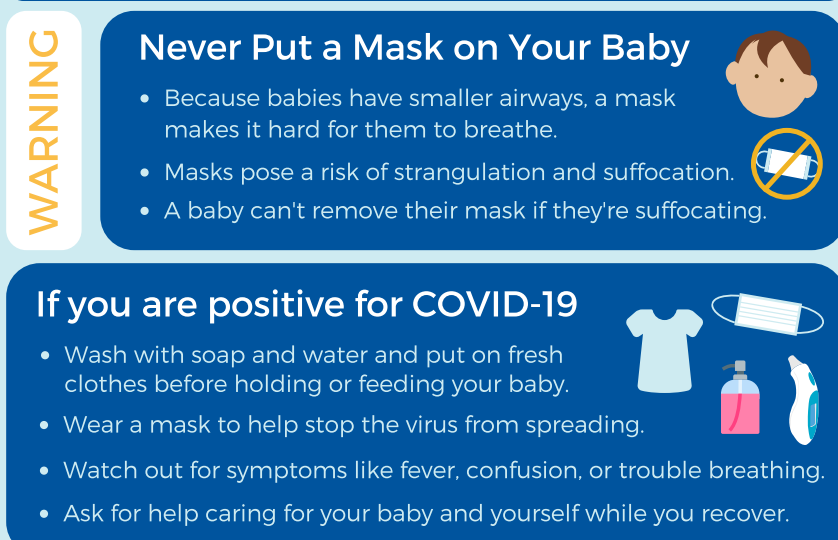

We can help protect each other. Learn more

www.nationalperinatal.org/COVID-19

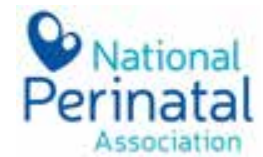

